\title{
An integrated quantitative structure and mechanism of action-activity relationship model of human serum albumin binding
}

Angela Serra ${ }^{1 \dagger}$, Serli Önlü ${ }^{1,4 \dagger}$, Pietro Coretto ${ }^{2}$ and Dario Greco ${ }^{1,3,5^{*}}$ (D)

\begin{abstract}
Background: Traditional quantitative structure-activity relationship models usually neglect the molecular alterations happening in the exposed systems (the mechanism of action, MOA), that mediate between structural properties of compounds and phenotypic effects of an exposure.

Results: Here, we propose a computational strategy that integrates molecular descriptors and MOA information to better explain the mechanisms underlying biological endpoints of interest. By applying our methodology, we obtained a statistically robust and validated model to predict the binding affinity to human serum albumin. Our model is also able to provide new venues for the interpretation of the chemical-biological interactions.
\end{abstract}

Conclusion: Our observations suggest that integrated quantitative models of structural and MOA-activity relationships are promising complementary tools in the arsenal of strategies aiming at developing new safe- and useful-bydesign compounds.

Keywords: QSAR, MOA, QSMARt, Molecular descriptors, Human serum albumin binding, Integrative analysis, Safe-bydesign, Lasso, Regression

\section{Introduction}

Quantitative structure-activity relationship (QSAR) models are increasingly applied in various fields, such as toxicity assessment and drug design [1]. QSAR models developed and validated in line with the Organization for Economic Co-Operation and Development (OECD) criteria [2] are recognized in silico tools for providing reliable activity data, bypassing long and laborious experimental assays. On the basis that structurally similar molecules have similar biological activities, classical QSAR models attempt to predict activity as a function of structural properties numerically defined as molecular descriptors (MDs) [1,3]. MDs provide extensive chemical information, such as presence and count of different sub-structures, functional groups, connectivity between

\footnotetext{
*Correspondence: dario.greco@tuni.fi

${ }^{\dagger}$ Angela Serra and Serli Önlü equally contributed to this work

${ }^{3}$ Institute of Biotechnology, University of Helsinki, Finland, Helsinki,

Finland

Full list of author information is available at the end of the article
}

atoms, topological and geometrical characteristics, which are relevant for predictive studies. Furthermore, 3D alignment-free molecular descriptors, based on two, three and four linear algebraic forms have been introduced to codify novel and orthogonal chemical information $[4,5]$.

Traditional QSAR models usually neglect the primary biological fingerprint of the exposure, consisting of the ensemble of molecular alterations happening at various cellular compartments of the exposed biological system, hereafter denoted as the mechanism of action (MOA). However, the relationship between structural properties and phenotypic effects of an exposure is indirectly mediated by its MOA. Systematically integrating MOA information, such as gene expression or external bioassay data, into QSAR modelling would expand our understanding of the chemical-biological interactions, hence paving the way to the development of the next generations of safeand useful-by-design compounds $[6,7]$. 
In the recent years, the implementation of omics technologies in toxicology studies has ignited the new field of toxicogenomics [8]. In this context, in depth molecular profiling opened new possibilities to outline the biosignature or MOA of exposures at an unprecedented granularity. However, to date, this information has been seldom utilized in combination with structural properties of the compounds to predict their effects [9-11].

Indeed, $\mathrm{Li}$ et al developed a methodology that jointly analyzes the chemical structural information and the gene expression profiles of cells treated by drugs. By means of a clustering methodology, they identified the most structurally similar sets of chemicals and the minimum set of genes related to chemical structural features [9]. Low et al. [10] used a machine learning methodology based on multiple nonlinear classifiers that integrates chemical descriptors and toxicogenomic data to classify drug molecules based on their hepatotoxicity (toxic/or non-toxic) effect in rats. Perualila-Tan et al. [11] proposed a statistical methodology that combines transcriptomic data and chemical information to predict a biological response by means of gene expression and infer if the response is caused by the presence or absence of a particular chemical sub-structure. These approaches are limited to binary classification problems (toxic/non toxic) and to the identification of correlations between MDs and MOA features. However, when modelling a continuous response variable, integrative regression models are a preferred option. Between the wide range of linear and nonlinear regression models, Lasso based methods have the advantage to generate easy to interpret models, since they automatically perform feature selection and have less parameter to be estimated as compared to nonlinear models, such as random forests, support vector regressors or neural networks.

Here, considering the OECD criteria [2], we propose a computational approach that combines MDs and MOA information to develop integrated quantitative structure and mechanism of action-activity relationship (QSMARt) models with the potential to better explain the role of specific structural properties in a bio-mechanistic way. To the best of our knowledge, the present study is the first report on an integrated QSMARt model to predict the binding affinity to HSA.

\section{Materials and methods}

\section{Dataset preparation}

Curated experimental binding affinity data of drug and drug-like molecules to HSA ( $\log K_{H S A}$; the binding constant obtained from the retention time on an immobilized HSA column using affinity chromatography) were obtained from [12]. All structures (as 3D SDF files) were retrieved from PubChem [13] and processed by the software DRAGON v. 7.0 [14] for the calculation of 5,325 MDs. An unsupervised feature reduction was applied to filter the constant $(>80 \%)$ and highly intercorrelated descriptors (pairwise correlation among all pairs of descriptors $>95 \%$ ) prior to training/test set splitting, and variable selection [15]. Thus, a data matrix comprising 1,198 MDs was generated (hereafter denoted as A). Transcriptomic data for drug treatments were retrieved from the Connectivity Map (CMap) build v2.0 repository [16]. Three human cell lines were available in the CMap project: prostate cancer (PC3), breast cancer (MCF7), and leukemia (HL60), respectively. The transcriptomic datasets were analyzed independently for each cell line. Raw data was imported into R v. 3.4 by using the justRMA function from the Bioconductor utilities [17] to annotate probes to Ensembl genes (by using the hthgu133ahsensgcdf (v. 22.0.0) annotation file from the brainarray website http://brainarray.mbni.med.umich .edu/), and to quantile normalize the resulting expression matrix. Next, the experimental batch effect due to technical variables was estimated and removed using the ComBat algorithm implemented in the sva package [18]. Linear models followed by eBayes pairwise comparisons [19] were performed to compute the log fold-change of each gene in each drug-control pairs. Of the 88 chemicals in the curated dataset [12], 59 were identified with reported gene expression data for at least two cell lines of the CMap dataset (MCF7 and PC3). The list of drugs used in this analysis is available in Additional file 1 . Consequently, two data matrices of log-fold changes for 11,868 genes in MCF7 (hereafter denoted as B) and PC3 cell lines (hereafter denoted as $\mathrm{C}$ ) were generated, respectively. Finally, MDs (A) and gene expression profiles (B and $\mathrm{C}$ ) were collated to create a single dataset (hereafter denoted as X) of 59 drugs and 24,934 features (1198 MDs and 11,868 genes for each cell line) for modeling the $\log K_{H S A}$.

\section{Modeling and validation}

QSMARt modeling was performed based on the lasso method [20] and power transformation of the MDs $(\alpha)$ and genes $(\gamma)$, respectively. $20 \%$ of the dataset X was kept as the test set and not used in the model selection phase. The remaining $80 \%$ of the data (training set) was further split 100 times in random training (90\%) and validation (10\%) sets by using a random split validation algorithm (RSVA). The splitting was performed based on the y-response variable, which was divided into three bins, from which the compounds are randomly assigned to train or test sets. Detailed methodology available as in Additional file 2. $\mathrm{R}$ scripts are available as Additional file 3. Next, the lasso method is used to fit a linear model to the training set for 100 different values of the lasso 
penalty estimated from the training matrix [21]. The lasso penalization value leading to the smallest mean squared error (MSE, $\lambda=0.166$ ), was considered (Additional file 4: Fig. S1). Only the features (MDs and/or genes) with nonzero coefficients were selected to derive the final model. Once the optimal features and parameters were identified, the entire training set was used to build the final model and the test set was only then used for external validation.

The following model was considered to predict the $\log K_{H S A}$ :

$$
y=X(\alpha, \gamma) \beta+\epsilon
$$

where, $X(\alpha, \gamma)$ is the matrix obtained by binding the matrices of $A(\alpha), B(\gamma)$, and $C(\gamma), A(\alpha)=\left(\left|a_{i j}\right|^{\alpha}\right)$, $B(\gamma)=\left(\left|b_{i j}\right|^{\gamma}\right), C(\gamma)=\left(\left|c_{i j}\right|^{\gamma}\right)$ (for $\alpha>0$ and $\left.\gamma>0\right), \beta$ is the vector of coefficients, and $\epsilon$ is the stochastic error, respectively.

The same power transformation $(\gamma)$ was used both for the MCF7 (B) and PC3 cell line (C). Considering $\alpha$ and $\gamma$ fixed, and $\beta$ the only structural/genomic parameter to be estimated, is conceptually equivalent to replacing the original sample measurements $X$ with $X(\alpha, \gamma)$. For fixed $\alpha$ and $\gamma$, the following lasso-type estimator is considered:

$$
\hat{\beta}=\operatorname{argmin}\|y-X(\alpha, \gamma) \beta\|_{2}^{2}+\lambda\|\beta\|_{1}
$$

where $\|\cdot\|_{2}$ is the euclidean norm, $\|\cdot\|_{1}$ is the $l^{1}$ norm and $\lambda$ is the lasso penalty.

The parameters $(\alpha, \beta, \gamma)$ were tuned to minimize the MSE on the training set. The RSVA was performed for a grid of nine distinct $\alpha$ and $\gamma$ values $(\alpha, \gamma=0.1,0.25,0.50,0.75,1,1.25,1.50,1.75,2)$ for all 81 possible pairs of $\left(\alpha_{i}, \gamma_{i}\right)$ with $i=1, \ldots, 81$. For each of the 81 combinations, the relevant set of features $f_{t}=\beta\left(\alpha_{t}, \gamma_{t}\right)$ (at $\left.t=1,2, \ldots, 81\right)$ associated with nonzero coefficients was identified, validated the 60 th percentile values of the distributions of the internal metrics computed on the multiple splits were considered), and used to train models on the whole training set. Next, the generated models were used to predict the $\log K_{H S A}$ on the test set. Following these steps, a population of candidate models was generated. Goodness of fit, robustness, and predictive performance of the candidate models were evaluated based on up-to-date internal and external validation parameters and criteria (Additional file 1) [22-28].

\section{Comparison with single view models}

In order to validate the QSMARt model, the same procedure was applied to the MDs and MOA features separately. The RSVA procedure was performed on nine $\alpha=0.1,0.25,0.50,0.75,1,1.25,1.50,1.75,2$ values for the MDs and nine $\gamma=0.1,0.25,0.50,0.75,1,1.25,1.50,1.75,2$ values for the MOA features. Furthermore, these two parameters, together with the $\lambda$ penalty, value were optimized independently for the MDs and MOA to identify the optimal setup that minimizes the MSE on the training set. These analyses led to 9 models for the MDs and 9 models for the MOA features. For each model, the relevant set of features, associated with non-zero coefficients, was identified and validated with the same approach described before. Goodness of fit, robustness, and predictive performance of the candidate models were evaluated based on up-to-date internal and external validation parameters and criteria (Additional file 1) [22-28]. In particular, distributions of the internal validation metrics computed with the RSVA procedure with 100 random splits were compared to identify which model overall give the better predictive performances.

\section{Applicability domain}

Based on the idea of consensus decision[29], different approaches were used to compute the applicability domain $(\mathrm{AD})$ of the identified models. In particular, $\mathrm{AD}$ was computed by means of the leverage method [30], the standardization approach [31], the euclidean [32] and city block distance methods [33], and the k-nearest neighbours method [34].

In the leverage method, the response outliers were determined as those with the predicted activity value $> \pm 3.0$ standardized residuals. The leverage value $(\mathrm{h})$ measures the distance from the centroid of the modeled space. A warning leverage (critical hat value, $h^{*}$ ) [30] was used to identify structural/MOA influential compounds ( $h>h^{*}$ denoting high-leverage chemicals). In a Williams plot [30], the leverage values were mapped against the standardized residuals to define the structural/MOA and the response spaces visually. Finally, the AD was reported as the percentile coverage for the training $\left(A D_{\text {Train }}\right)$ and test $\left(A D_{\text {Test }}\right)$ set, respectively. Moreover, the Insubria graph [15] of leverage values against calculated/predicted activity values was used to visualize the interpolated ( $h<h^{*}$ denoting chemicals inside the structural/MOA $\mathrm{AD}$ of the training set) and extrapolated $\left(h>h^{*}\right.$ characterizing chemicals outside the structural/MOA AD) predictions for all the datasets considered in this study. In this case, the response $\mathrm{AD}$ was the prediction range of the model.

The standarization approach [31] is based on the assumption that in case of normal distribution, $99.7 \%$ of the population will remain within the range mean \pm 3.0 standard deviation (SD). Thus, all molecular descriptors are first standardized. Afterwards, any compound outside this zone is dissimilar to the rest and majority of the compounds. Thus, if the standardized value for descriptor $i$ of compound $k$ is more than 3 , then the compound 
should be an X-outlier (if in the training set) or outside $\mathrm{AD}$ (if in the test set) based on descriptor $i$.

In the distance based methods [32-34], the distance between the chemical and the center of the training data set is computed. The threshold, for both Euclidean and City block distances, is the largest distance between the training set data points and the center of the training data set. Furthermore, the distance between the test samples and the center of the dataset is computed. The test points with a distance greater than the computed threshold are considered outliers. The $\mathrm{AD}$ was reported as the percentile coverage of the test set $\left(A D_{\text {Test }}\right)$.

In the k-nearest neighbours method [34], the distance between every train compound and its k-nearest neighbours in the training set is computed. A threshold is calculated as the largest of these distances. Subsequently, the distance between every test compounds and its $k$-nearest neighbours in the training set is computed. If the calculated distance values of test set compounds is within the defined threshold, then the prediction of these compounds are considered to be reliable. In this method the $\mathrm{k}$ value was set to 3 . The $\mathrm{AD}$ was reported as the percentile coverage of the test set $\left(A D_{\text {Test }}\right)$.

The final consensus value on the training compounds is computed as a mean of the leverage and standardization methods, while the consensus on the test set is computed as the mean of all the different approaches.

\section{Selection of the final model}

Among the generated candidate models, the one with the best compromise between statistical robustness, predictive performance, widest $\mathrm{AD}$, and smallest dimension was selected as the final model. To this end, all 81 alternative specifications were filtered based on multiple up-to-date statistical acceptance criteria (highlighted in Additional file 1). Only the models both satisfying the internal and external validation requirements, and providing $100 \% A D_{\text {Test }}$ coverage, with the consensus method, were considered eligible. Moreover, the transformation parameters $\left(\alpha^{*}, \gamma^{*}\right)$ achieving the best predictive performance were selected as the set of indices of eligible solutions by solving $\left(\alpha^{*} \gamma^{*}\right)=\operatorname{argmin}_{t}\left(E\left(\alpha_{t}, \gamma_{t}\right)\right.$; $t \in I)$ with $I \subseteq\{1, \ldots, 81\}$. Finally, the model satisfying all eligibility criteria, consisting of the smallest number of structural/MOA features, and with the widest $A D_{\text {Train }}$ coverage was selected as the ultimate model.

\section{Application of the final model}

The optimal model was applied to a set of external compounds for which the $\log K_{H S A}$ is unavailable. To this purpose, an independent set of 799 drugs from the CMap dataset [16] with gene expression data available on both MCF7 and PC3 cell lines was considered. SDF files for these compounds were retrieved from PubChem [13] and fed into DRAGON v. 7.0 [14] to generate molecular descriptors. Gene expression data was preprocessed similarly to the dataset of 59 compounds, as described above. The list of drugs in the external dataset is available in Additional file 1. The TSNE projection technique [35] was used to visualize the distribution of the albumin and the external datasets based on the six MDs/MOA features of the QSMARt model as well as the three MOA features and three MDs.

\section{Results and discussion}

QSMARt predictive model for the binding affinity to HSA

Here, we built an integrated model (QSMARt) comprising molecular descriptors and MOA features to predict binding affinity to human serum albumin. To this end, we derived 81 candidate models by applying a Lasso penalty parameter optimisation. The 81 models and their evaluation metrics are reported in Additional file 1. The full lists of selected MDs and genes, along with their occurrence frequencies, are available in Additional file 1. Upon rigorous evaluation based on the OECD validation principles [2], we selected a final model of six structural/MOA features: three molecular descriptors and three gene expression patterns (Eq. 3).

$$
\begin{aligned}
& \log _{H S A}=-0.372+0.012 \mid \text { Mor }\left.23 i\right|^{1.25} \\
& \quad-0.042|N-072|^{1.25}+0.139 \mid \text { ALOGP }\left.\right|^{1.25} \\
& \quad-2.980 \mid \text { MCF7_ENSG00000112115 }\left.\right|^{1.75} \\
& -0.075 \mid \text { PC3_ENSG00000197646 }\left.\right|^{1.75} \\
& -0.216 \mid \text { PC3_ENSG00000276644 }\left.\right|^{1.75}
\end{aligned}
$$

A good concordance between the predicted and experimental data is shown in Fig. 1. Our model fulfils the criteria regarding the goodness of fit and the internal and

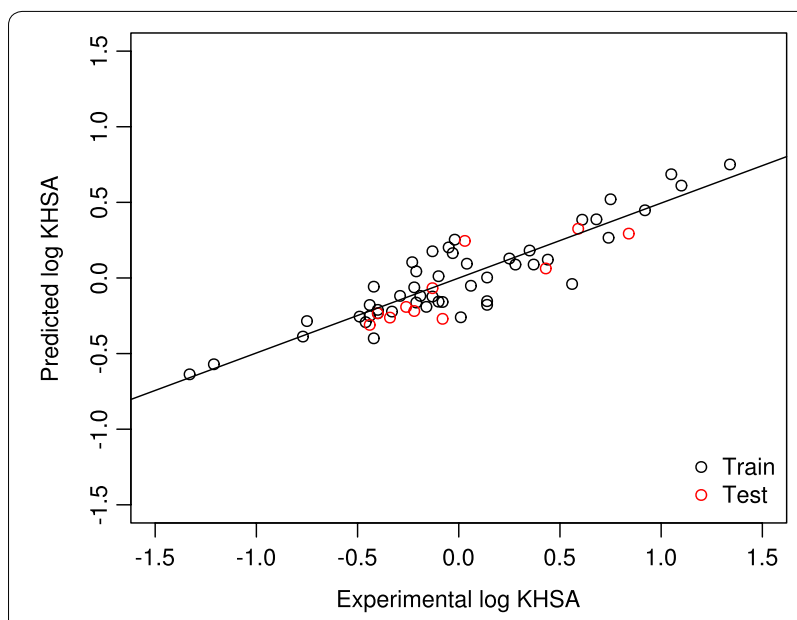

Fig. 1 Predicted log $K_{H S A}$ versus experimental log $K_{H S A}$ values of training set (black) and test set (red) chemicals 
external validation requirements, as shown in Additional file 1 . Moreover, the final hybrid model statistics passed all the recommended thresholds except the CCC metric (Additional file 1). Indeed, the $Q_{L 10-O u t}, R_{t r}^{2}, R_{t e}^{2}, Q_{F_{1}}^{2}, Q_{F_{2}}^{2}$, $Q_{F_{3}}^{2}$ are greater than 0.6 , but, the $C C C_{T e}$ value is smaller than 0.85 . Next, we defined the AD of our model based on the consensus strategy. Noteworthily, all chemicals of the test set are inside the $\mathrm{AD}$ spaces (Additional file 1), suggesting that all the predictions were reliably interpolated. For visualization purposes we show the AD computed by means of the the leverage approach [30] in Fig. 2.

\section{Impact of the integration approach and comparison to sub-models}

Next, in order to evaluate the impact of the integration strategy, we compared our QSMARt integrated final model with the two obtained by applying our approach to the MDs and genes separately. We ran the SVA methodology for the same nine $\alpha$ and $\gamma$ values and we obtained 9 models for the MDs, while only two models were obtained by using the genes alone, since no fitting was obtained for $7 \gamma$ values. The best models for the MDs and genes respectively are the following:

$$
\begin{aligned}
\log _{H S A}= & -0.335+0.077 \mid \text { Mor }\left.23 i\right|^{0.11}-0.012|R 8 s .|^{0.11} \\
& +0.007|C .040|^{0.11}-0.061|N-072|^{0.11} \\
& +0.062|A L O G P|^{0.11}-0.003\left|C A T S 3 D_{0} 6_{A} P .\right|^{0.11} \\
& +0.0006 \mid \text { piPC08 }\left.\right|^{0.11}-0.010|G A T S 2 i|^{0.11} \\
& +0.001 \mid \operatorname{SpMax}_{B} \text { h.v. }\left.\right|^{0.11}
\end{aligned}
$$

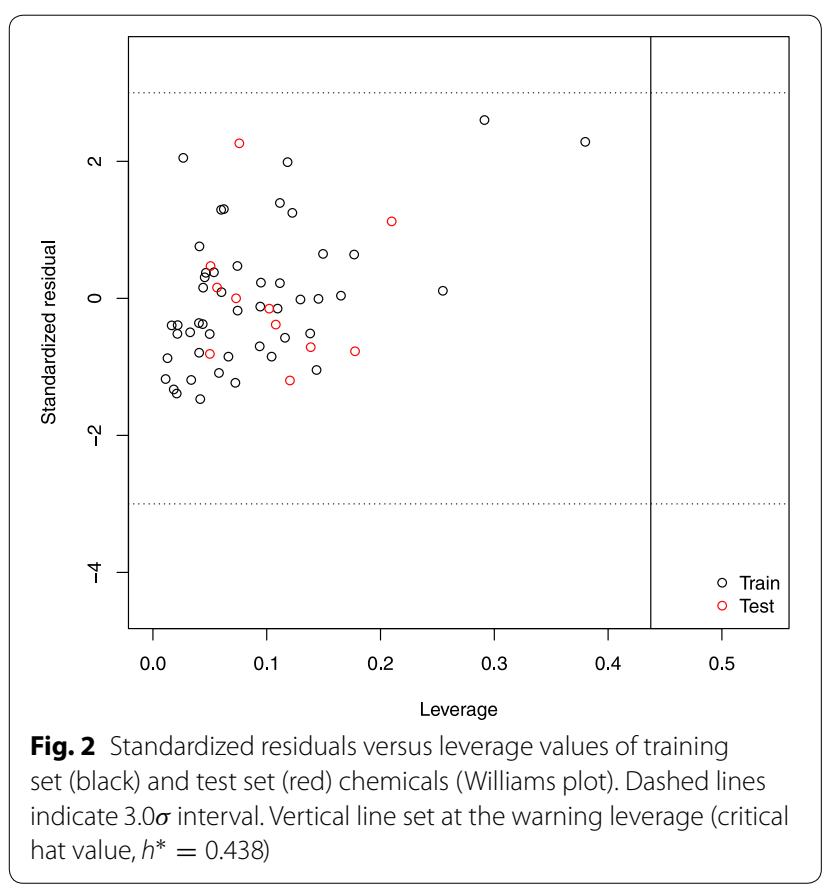

$$
\begin{aligned}
& \log K_{H S A}=0.042+3.84\left|M C F 7 \_E N S G 00000185950\right|^{0.16} \\
& \text { - 11.163|MCF7_ENSG00000112115 }\left.\right|^{0.16} \\
& -0.758\left|M C F 7 \_E N S G 00000135100\right|^{0.16} \\
& +0.193 \mid \text { PC3_ENSG00000128228 }\left.\right|^{0.16} \\
& +0.0007\left|P C 3 \_E N S G 00000168209\right|^{0.16} \\
& +0.040 \mid \text { PC3_ENSG00000110619| }\left.\right|^{0.16} \\
& -0.755\left|P C 3 \_E N S G 00000064687\right|^{0.16} \\
& -1.310 \mid \text { PC3_ENSG00000168875| }\left.\right|^{0.16} \\
& -9.301\left|P C 3 \_E N S G 00000276644\right|^{0.16}
\end{aligned}
$$

As evidenced in Additional file 1, the QSMARt model is characterized by overall better values of all the relevant diagnostic statistics. This analysis, hence, highlighted an overall better statistical performance of the integrated QSMARt model (Eq. 3) over the two competitor models (Eq. 4 and Eq. 5). In particular, the QSMARt model consists of less features, since it uses only 3 MDs and 3 genes, while the other two models use 9 MDs and 9 genes, respectively. Furthermore, the model coming from the genes does not show any predictive capability on the test set $\left(R_{\text {test }}^{2}=0.10\right)$ although its $R_{\text {train }}^{2}=0.61$. On the other hand the model obtained by using only MDs has good predictive capabilities, even thought they are smaller than the one obtained by the QSMARt model. Furthermore, when comparing the distributions of the $Q^{2}, Q^{2} F_{1}$ $, Q^{2} F_{2}, Q^{2} F_{3}$ and $C C C$ metrics that are computed with the RSVA method, the performances of the QSMARt model are better than those of the other two models (Additional file 5: Fig. S2).

\section{Mechanistic interpretation of the features included in the QSMARt model}

Mechanistic interpretation of the molecular descriptors included in a model is an OECD principle of QSAR validation [2]. The hybrid model was built with the following MDs: Mor23i, N-072, and ALOGP. Mor23i is a measure of the pair-wise interatomic distance and ionization potential [36]. Ionization potential is the amount of energy required to extract one electron from a chemical system, i.e., a measure of the capability of a molecule to give the corresponding cation. Mor23i and $\log K_{H S A}$ are positively correlated (Eq. 3), implying that the higher the ionization potential, the higher the HSA binding affinity. This further suggests that electron-pair acceptors (Lewis-acids) have higher binding affinity to HSA. Given the positive coefficient of Mor23i in our model equation, compounds with more acidic properties have higher binding affinity to HSA. On the other hand, due to the mathematical background, the distance between two influential atoms may majorly define the descriptor [36]. 
Therefore, a more detailed interpretation could be useful for molecular design purposes.

$\mathrm{N}-072$ is a descriptor counting the nitrogen-centered fragments of $\mathrm{RCO}-\mathrm{N}<$ or $>\mathrm{N}-\mathrm{X}=\mathrm{X}$ in a chemical structure, where $\mathrm{R}$ is any group bound through carbon, $\mathrm{X}$ is any electronegative atom, such as oxygen, nitrogen, sulfur, phosphorus, and halogens, - is single and = is double bonds, respectively [3]. The negative coefficient in the final model indicates that chemicals with $\mathrm{N}-072$ fragments show less affinity to HSA binding. Similarly, N-072 was reported elsewhere as affecting the relative fluorescence intensity ratio [37].

ALOGP is a measure of hydrophobicity as the logarithm of n-octanol/water partition coefficient. Based on the Ghose-Crippen method [38], it is calculated as the summation of atomic contributions to overall molecular hydrophobicity. Clearly, having a positive coefficient in the model equation, ALOGP explains an increased affinity to HSA binding. It has been already reported in relation to binding affinity to HSA [12, 39]. Furthermore, earlier studies on the crystallographic structure of HSA and binding affinity evidenced that the binding sites of HSA are mainly composed of hydrophobic residues, further revealing that hydrophobicity is a major property encoding the binding affinity, as reviewed in [40, 41].

Three genes are included in the final QSMARt model, namely Interleukin 17A (IL17A) from the MCF7, Programmed Cell Death 1 Ligand 2 (PD-L2), and Dachshund Family Transcription Factor 1 (DACH1) from the PC-3 transcriptomic datasets, respectively. The expression of IL17A is documented in the MCF7 cell line, where it has been tested as target of chemotherapeutic strategies aiming at altering autophagic ability of breast cancer cell lines [42]. Alteration of the expression of PD-L2, a ligand of PD-1, has been observed both in prostate cancer in response to anti-PD-1 therapy [43]. DACH1 is a transcription factor expressed in prostate cancer, where its low expression is associated with higher malignant potential [44]. Interestingly, all these three genes have known immunomodulatory properties, either as proinflammatory (IL17A) or immunosuppressive (PD-L2 and DACH1). Since their QSMARt model coefficients are negative, the impact of drugs to alter their expression is inversely proportional to HSA binding affinity. These results, for instance, suggest that the serum supplementation in the cell culture medium and the compound dosages should be mutually adjusted when testing drugs in vitro, such as in the CMap experiments.

Next, we considered the correlation between the three gene expression patterns and the three MDs included in the QSMARt model (Fig. 3). All the genes in the model were negatively correlated with Mor23i and ALOGP, and positively correlated with $\mathrm{N}-072$, respectively. These

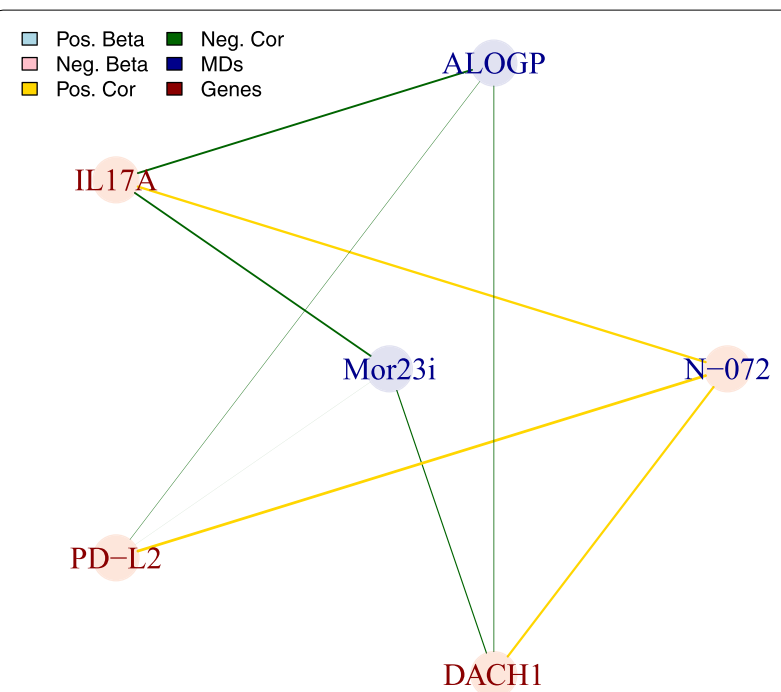

Fig. 3 Correlation graph of the six MDs/MOA features of the QSMARt model. Vertex color represent the sign of the associated beta value while edge colors show the sign of the correlation of the features across the $X$ dataset

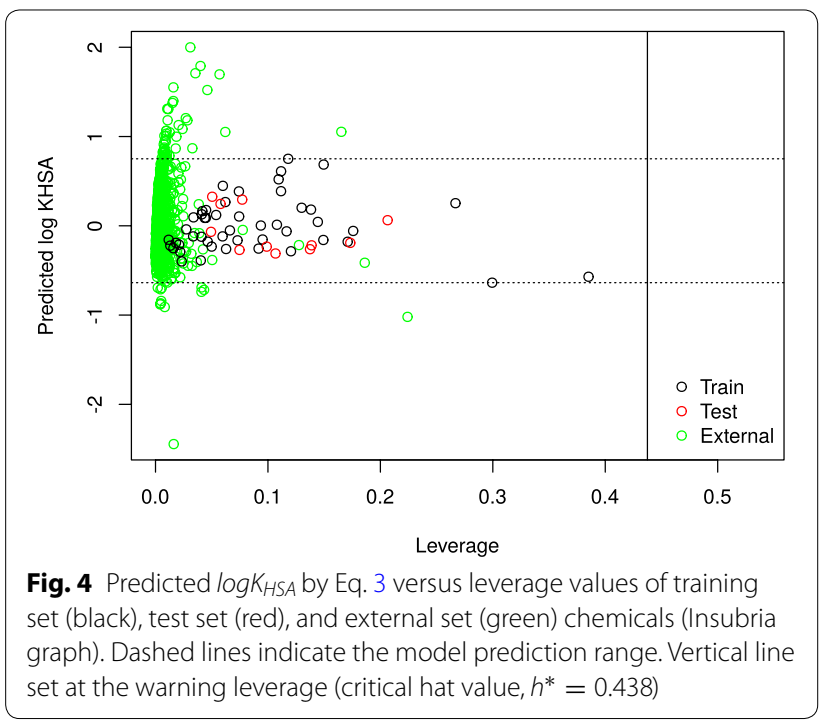

results imply that potentially less acidic (lower values of Mor23i) and less lipophilic compounds (lower values of ALOGP) have a higher impact in altering the expression of these three genes.

Altogether, according to the QSMARt model, compounds with higher values of ionization potential and hydrophobicity, and less nitrogen-centered residuals, as well as lower expression alteration of the immunomodulatory genes IL17A, PD-L2 and DACH1, have higher binding affinity to HSA. 
Taken together, these results provide an extended mechanistic interpretation of the interactions of chemicals and biological systems by providing direct associations between specific structural and biological properties of the exposure.

\section{Application of the QSMARt model}

Finally, we tested the performance of our QSMARt model in predicting the $\log K_{H S A}$ for an independent set of 799 compounds extracted from the CMap dataset. With 741 chemicals in the $\mathrm{AD}$, our model provided a remarkable prediction coverage of $93 \%$ (Fig. 4). It is noteworthy to emphasize that no external chemicals falling outside the structural/MOA feature domain were identified.
However, 58 drugs appeared outside the model prediction range and were further investigated. For this, we inspected the distribution of the different subsets of compounds in a projected space based on the six MDs/ MOA features of the QSMARt model (Fig. 5a) as well as the three MOA features (Fig. 5b) and three MDs (Fig. 5c) considered separately. This analysis evidenced that the external set chemicals falling outside the model prediction range show less structural commonalities with the rest of the compounds (Fig. 5c) but are genomically confounded with the others (Fig. 5b). Thus, we further investigated the value of the MDs for the external dataset and, as we can see from Fig. 5d, drugs falling outside the prediction range of our model have higher value for the ALOGP MD.
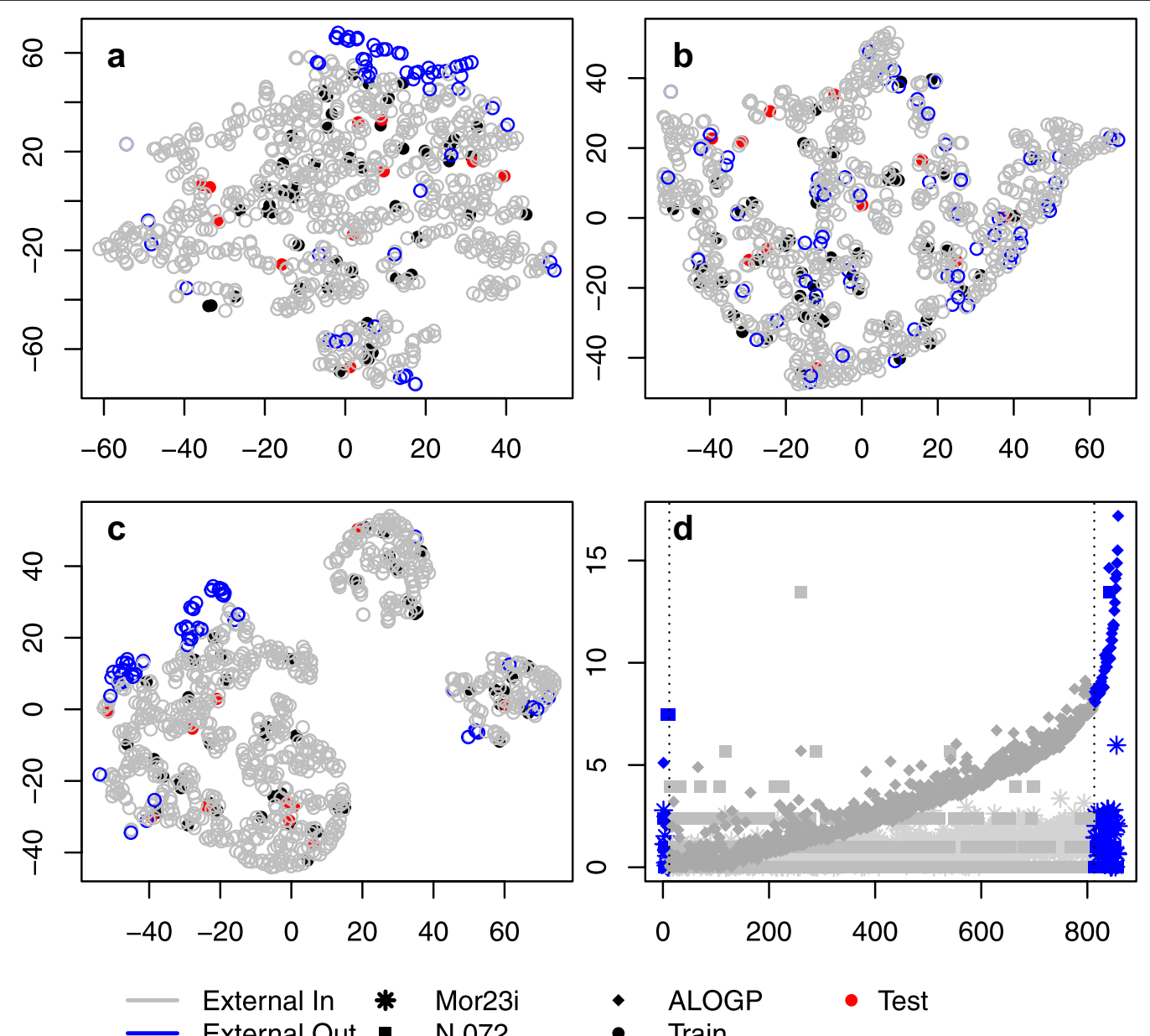

Fig. 5 TSNE projection of the drugs in the albumin and external dataset. The projection was performed by using the set of genes and MDs (a), only the genes (b) and only the MDs (c) in the optimal hybrid model. The outliers are in the border area of the dataset for the molecular descriptors (c), while they are similar to the rest of the external set fort the gene log-fold change (b). Likewise, the outliers still appear on the border for the combined two sets of features (a). In panel (d) the values of the three MDs is plotted ( $y$ axis) for the drugs in the albumin and external dataset $(x$ axis). The drugs are ordered based on their predicted log $K_{H S A}$ value. Drugs from the external set that falls in the model prediction range are marked in gray, while the ones that are outside the range are marked in blue. Drugs in the training set are marked in black while drugs in the test set are marked in red 


\section{Biological relevance of the QSMARt model}

In order to better understand the possible impact of the QSMARt model, we investigated its performance on drugs grouped by the ATC (Anatomical, Therapeutic, Chemical) code system as defined by the World Health Organization (WHO) [45]. The ATC codes classify the drugs into different groups in accordance with the organ or system on which they act and their chemical, pharmacological, and therapeutic properties. We performed our analyses by considering the anatomical subgroup (level 1) and the therapeutic subgroup (level 2) of the ATC codes. We investigated the relationship between the experimental $v s$. predicted logKHSA values, of the 59 drugs present in our dataset, and their grouping in ATC level 1 and 2 (Additional file 6: Fig. S3). This analysis highlights that the two drugs cefuroxime and amoxicillin, belonging to the ATC class J (any-inflammatory), show the lowest range of experimental and predicted logKHSA. Likewise, a large group of ATC class C compounds (cardiovascular system) are in the mid range of the distribution, while four ATC class $N$ (nervous system) are grouped in the highest range of the experimental/predicted logKHSA. Next, we inspected the larger set of 799 drugs used for the external validation, for which no experimental value of $\operatorname{logKHSA}$ was available. In this case, we looked at the distribution of the predicted logKHSA values in the level 1 and level 2 ATC codes (Additional files 7: Fig. S4 and 8: Fig. S5). Also, this analysis shows that the compounds belonging to the ATC class J (anti-inflammatory) have the lowest levels of predicted logKHSA. On the opposite, drugs of the ATC class A (digestive system), G (genitourinary system) and $\mathrm{N}$ (nervous system) have the highest predicted $\operatorname{logKHSA}$. These results confirm our observations on the 59 drugs present in our discovery set.

The genes selected in our model are involved in several signalling pathways, especially in cancer and immune signalling. Thus, we investigated their expression values between immunomodulatory and non immunomodulatory compounds. We identified the level 2 classes L03 and L04 to be immunostimulant and immunosuppressant, respectively. Unfortunatelly, none of the compounds available in the Connectivity map data set belong to the class L03, while four are annotated as L04. To perform the comparison, we selected the compound structurally least similar to each of the L04 drugs in the Connectivity Map dataset, and plotted the respective expression values for each of the three genes included in our final model (Additional file 9: Fig. S6). While MCF7_ENSG00000112115 And PC3_ENSG00000197646 did not show any difference, the gene PC3_ENSG00000276644 showed a trend with higher expression in L04 drugs as compared to their least similar ones.

\section{Conclusion}

In this study, we proposed a computational strategy to define quantitative models of structural and mechanism of action-activity relationships (QSMARt). Moreover, we investigated the effectiveness of hybrid QSMARt model comprising both MDs and MOA information to better explain the biological mechanisms underlying endpoints of interest. We applied our methodology to predict human serum albumin (HSA) binding, obtaining a statistically robust and validated model that provides new venues for the interpretation of the chemical-biological interactions. QSMARt models are promising complementary tools to develop new safe- and useful-by-design compounds.

\section{Additional file}

\begin{abstract}
Additional file 1. This file contains the following supplementary tables: Table S1: Dataset; Table S2: Parameters and criteria considered for goodness of fit, internal and external validation; Table S3: External dataset with predicted LogK hai Table S4: Molecular descriptors appearing in the models; Table S5: Genes appearing in the models; Table S6: Summary of the models parameters and evaluation metrics.
\end{abstract}

Additional file 2. This file contains the formal descriptions of the methodology and the RSVA algorithm

Additional file 3. File containing the $\mathrm{R}$ functions and scripts to create the integrative model.

Additional file 4. Fig. S1. Estimation of the optimal $\lambda$; value with the RSVA algorithm.

Additional file 5. Fig. S2. Comparison of the model validation curves.

Additional file 6. Fig. S3. Scatterplot of experimental vs. predicted $\log _{\text {HSA }}$ values of the 59 drugs, coloured by ATC codes level 1 and 2 .

Additional file 7. Fig. S4. Boxplot of the predicted logK $\mathrm{K}_{\mathrm{HSA}}$ values of the 799 external compounds coming from CMap dataset grouped by ATC codes level 1.

Additional file 8. Fig. S5. Boxplot of the predicted log $K_{H S A}$ values of the 799 external compounds coming from CMap dataset grouped by ATC codes level 2.

Additional file 9. Fig. S6. Boxplot of the expression values of the three selected genes, grouped by immunosuppressant and their less similar drugs.

\section{Abbreviations}

AD: applicability domain; CMap: connectivity map; HL60: leukemia cell line; HSA: human serum albumin; Lasso: least absolute shrinkage and selection operator; MCF7: breast cancer cell line; MDs: molecular descriptors; MOA: mechanism of action; OECD: organization for economic co-operation and development; PC3: prostate cancer cell line; QSAR: quantitative structureactivity relationship; QSMARt: quantitative structure and mechanism of actionactivity relationship.

\section{Authors' contributions}

AS developed and implemented the method, analysed the data, wrote the manuscript; SÖ evaluated and interpreted the results, wrote the manuscript; PC developed the method and wrote the manuscript; DG conceived and supervised the project, interpreted the results, wrote the manuscript. All authors read and approved the final manuscript. 


\section{Funding}

This study was supported by the Academy of Finland (grant agreements 275151 and 292307).

\section{Availability of data and materials}

The datasets supporting the conclusions of this article are included within the article as Additional Files.

\section{Competing interests}

The authors declare that they have no competing interests.

\section{Author details}

${ }^{1}$ Faculty of Medicine and Health Technology, Tampere University, Arvo Ylpön katu 34, Tampere, Finland. ${ }^{2}$ DISES, STATLAB, University of Salerno, Giovanni Paolo II 132, Fisciano, Italy. ${ }^{3}$ Institute of Biotechnology, University of Helsinki, Finland, Helsinki, Finland. ${ }^{4}$ Present Address: Corporate Product Safety/Henkel AG \& Co. KGaA, Düsseldorf, Germany. ${ }^{5}$ BioMediTech institute, Tampere University, Tampere, Finland.

Received: 16 January 2019 Accepted: 22 May 2019 Published online: 06 June 2019

\section{References}

1. Cherkasov A, Muratov EN, Fourches D, Varnek A, Baskin II, Cronin M, Dearden J, Gramatica P, Martin YC, Todeschini R, Consonni V, Kuz'min VE, Cramer R, Benigni R, Yang C, Rathman J, Terfloth L, Gasteiger J, Richard A Tropsha A (2014) QSAR modeling: where have you been? where are you going to? J Med Chem 57(12):4977-5010. https://doi.org/10.1021/jm400 4285

2. OECD (2014) Guidance document on the validation of (quantitative) structure-activity relationship [(Q)SAR] models, p 154. https://www.oecdilibrary.org/content/publication/9789264085442-en. Accessed 12 Mar 2018

3. Todeschini R, Consonni V. (eds.): Molecular descriptors for chemoinformatics: volume I: alphabetical listing/volume II: appendices, references. methods and principles in medicinal chemistry. Wiley-VCH Verlag GmbH \& Co. KGaA, Weinheim, Germany (2009). https://doi.org/10.1002/97835 27628766. Accessed 12 Mar 2018

4. García-Jacas CR, Contreras-Torres E, Marrero-Ponce Y, Pupo-Meriño M, Barigye SJ, Cabrera-Leyva L (2016) Examining the predictive accuracy of the novel $3 \mathrm{~d}$ n-linear algebraic molecular codifications on benchmark datasets. J Cheminform 8(1):10

5. Valdés-Martiní JR, Marrero-Ponce Y, García-Jacas CR, Martinez-Mayorga K, Barigye SJ, d'Almeida YSV, Pérez-Giménez F, Morell CA, et al. (2017) Qubils-mas, open source multi-platform software for atom-and bondbased topological (2d) and chiral (2.5 d) algebraic molecular descriptors computations. J Cheminform 9(1):35 (2017)

6. Chen Q, Wu L, Liu W, Xing L, Fan X (2013) Enhanced QSAR model performance by integrating structural and gene expression information. Molecules 18(9):10789-10801. https://doi.org/10.3390/molecules180910 789

7. Wang W, Kim MT, Sedykh A, Zhu H (2015) Developing enhanced bloodbrain barrier permeability models: Integrating external bio-assay data in QSAR modeling. Pharm Res 32(9):3055-3065. https://doi.org/10.1007/ s11095-015-1687-1

8. Alexander-Dann B, Pruteanu LL, Oerton E, Sharma N, Berindan-Neagoe I, Módos D, Bender A (2018) Developments in toxicogenomics: understanding and predicting compound-induced toxicity from gene expression data. Mol Omics 14(4):218-236. https://doi.org/10.1039/c8mo0 0042e

9. Li Y, Tu K, Zheng S, Wang J, Li Y, Hao P, Li X (2011) Association of feature gene expression with structural fingerprints of chemical compounds. J Bioinform Comput Biol 9(4):503-519

10. Low Y, Uehara T, Minowa Y, Yamada H, Ohno Y, Urushidani T, Sedykh A, Muratov E, Kuz'min V, Fourches D, Zhu H, Rusyn I, Tropsha A (2011) Predicting drug-induced hepatotoxicity using QSAR and toxicogenomics approaches. Chem Res Toxicol 24(8):1251-1262. https://doi.org/10.1021/ tx200148a
11. Perualila-Tan N, Kasim A, Talloen W, Verbist B, Göhlmann HWH, Consortium Q, Shkedy Z (2016) A joint modeling approach for uncovering associations between gene expression, bioactivity and chemical structure in early drug discovery to guide lead selection and genomic biomarker development. Stat Appl Genet Mol Biol 15(4):291-304. https:// doi.org/10.1515/sagmb-2014-0086

12. Önlü S, Türker Sacan M (2017) Impact of geometry optimization methods on QSAR modelling: a case study for predicting human serum albumin binding affinity. SAR QSAR Environ Res 28(6):491-509. https://doi. org/10.1080/1062936X.2017.13432

13. Kim S, Thiessen PA, Bolton EE, Chen J, Fu G, Gindulyte A, Han L, He J, He S, Shoemaker BA, Wang J, Yu B, Zhang J, Bryant SH (2016) PubChem substance and compound databases. Nucleic Acids Res 44(D1):1202-13. https://doi.org/10.1093/nar/gkv951

14. Mauri A, Consonni V, Pavan M, Todeschini R (2006) Dragon software: an easy approach to molecular descriptor calculations. Match 56(2):237-248

15. Gramatica P, Chirico N, Papa E, Cassani S, Kovarich S (2013) Qsarins: a new software for the development, analysis, and validation of QSAR mlr models. J Comput Chem 34(24):2121-2132

16. Lamb J, Crawford ED, Peck D, Modell JW, Blat IC, Wrobel MJ, Lerner J, Brunet J-P, Subramanian A, Ross KN, Reich M, Hieronymus H, Wei G, Armstrong SA, Haggarty SJ, Clemons PA, Wei R, Carr SA, Lander ES, Golub TR (2006) The connectivity map: using gene-expression signatures to connect small molecules, genes, and disease. Science 313(5795):1929-1935. https://doi.org/10.1126/science.1132939

17. Irizarry RA, Bolstad BM, Collin F, Cope LM, Hobbs B, Speed TP (2003) Summaries of affymetrix GeneChip probe level data. Nucleic Acids Res 31(4):15. https://doi.org/10.1093/nar/gng015

18. Leek J, Johnson W, Parker H, Jaffe A, Storey J (2014) SVA: surrogate variable analysis $\mathrm{R}$ package version 3.10.0

19. Ritchie ME, Phipson B, Wu D, Hu Y, Law CW, Shi W, Smyth GK (2015) limma powers differential expression analyses for RNA-sequencing and microarray studies. Nucleic Acids Res 43(7):47. https://doi.org/10.1093/nar/gkv00

20. Tibshirani R (1996) Regression shrinkage and selection via the lasso. J R Stat Soc Ser B (Methodol) 58:267-288

21. Friedman J, Hastie T, Tibshirani R (2010) Regularization paths for generalized linear models via coordinate descent. J Stat Softw 33(1):1-22. https ://doi.org/10.18637/jss.v033.i01

22. Golbraikh A, Tropsha A (2002) Beware of q2!. J Mol Graph Model 20(4):269-276. https://doi.org/10.1016/S1093-3263(01)00123-1

23. Aptula AO, Jeliazkova NG, Schultz TW, Cronin MT (2005) The better predictive model: high q2 for the training set or low root mean square error of prediction for the test set? QSAR Comb Sci 24(3):385-396

24. Shi LM, Fang H, Tong W, Wu J, Perkins R, Blair RM, Branham WS, Dial SL, Moland CL, Sheehan DM (2001) OSAR models using a large diverse set of estrogens. J Chem Inf Comput. Sci 41(1):186-195

25. Chirico N, Gramatica P (2012) Real external predictivity of QSAR models part 2. new intercomparable thresholds for different validation criteria and the need for scatter plot inspection. J Chem Inf Model 52(8):20442058. https://doi.org/10.1021/ci300084j

26. Schüürmann G, Ebert R-U, Chen J, Wang B, Kühne R (2008) External validation and prediction employing the predictive squared correlation coefficient test set activity mean vs training set activity mean. J Chem Inf Model 48(11):2140-2145. https://doi.org/10.1021/ci800253u

27. Consonni V, Ballabio D, Todeschini R (2009) Comments on the definition of the q2 parameter for QSAR validation. J Chem Inf Model 49(7):1669_ 1678. https://doi.org/10.1021/ci900115y

28. Consonni V, Ballabio D, Todeschini R (2010) Evaluation of model predictive ability by external validation techniques. J Chemom 24(3-4):194-201. https://doi.org/10.1002/cem.1290

29. García-Jacas CR, Martinez-Mayorga K, Marrero-Ponce Y, Medina-Franco $J$ (2017) Conformation-dependent qsar approach for the prediction of inhibitory activity of bromodomain modulators. SAR QSAR in Environ Res 28(1):41-58

30. Gramatica P (2007) Principles of qsar models validation: internal and external. Mol Inform 26(5):694-701

31. Roy K, Kar S, Ambure P (2015) On a simple approach for determining applicability domain of qsar models. Chemom Intell Lab Syst 145:22-29 
32. Jaworska J, Nikolova-Jeliazkova N, Aldenberg T (2004) Review of methods for applicability domain estimation. Report. The European CommissionJoint Research Centre, Ispra, Italy

33. CDATA-Hair J, Anderson R, Tatham R, Black W (1998) Multivariate Data Analysis. Prentice Hall, Englewood Cliffs, N

34. Sheridan RP, Feuston BP, Maiorov VN, Kearsley SK (2004) Similarity to molecules in the training set is a good discriminator for prediction accuracy in qsar. J Chem inf Comput Sci 44(6):1912-1928

35. Maaten LVD, Hinton G (2008) Visualizing data using t-SNE. J Mach Learn Res 9:2579-2605

36. Devinyak O, Havrylyuk D, Lesyk R (2014) 3d-morse descriptors explained. J Mol Graph Model 54:194-203

37. Xu J, Xiong Q, Chen B, Wang L, Liu L, Xu W (2009) Modeling the relative fluorescence intensity ratio of eu(III) complex in different solvents based on QSPR method. J Fluoresc 19(2):203-209. https://doi.org/10.1007/ s10895-008-0403-5

38. Ghose AK, Crippen GM (1987) Atomic physicochemical parameters for three-dimensional-structure-directed quantitative structure-activity relationships. 2. Modeling dispersive and hydrophobic interactions. J Chem Inf Ccomput Sci 27(1):21-35

39. Chen L, Chen X (2012) Results of molecular docking as descriptors to predict human serum albumin binding affinity. J Mol Graph Model 33:35-43

40. Colmenarejo G (2003) In silico prediction of drug-binding strengths to human serum albumin. Med Rese Revi 23(3):275-301
41. Lambrinidis G, Vallianatou T, Tsantili-Kakoulidou A (2015) In vitro, in silico and integrated strategies for the estimation of plasma protein binding: A review. Adv Drug Deliv Rev 86:27-45

42. Garbar C, Mascaux C, Giustiniani J, Merrouche Y, Bensussan A (2017) Chemotherapy treatment induces an increase of autophagy in the luminal breast cancer cell MCF7, but not in the triple-negative MDA-MB231. Sci Rep 7(1):7201

43. Taube JM, Klein AP, Brahmer JR, Xu H, Pan X, Kim JH, Chen L, Pardoll DM, Topalian SL, Anders RA (2014) Association of PD-1, PD-1 ligands, and other features of the tumor immune microenvironment with response to anti-PD-1 therapy. Clin Cancer Res 3271

44. Wu K, Yuan X, Pestell R (2015) Endogenous dach1 in cancer. Oncoscience 2(10):803

45. Organization WH et al (2006) Who collaborating centre for drug statistics methodology: atc classification index with ddds and guidelines for atc classification and ddd assignment. Norwegian Institute of Public Health, Oslo, Norway

\section{Publisher's Note}

Springer Nature remains neutral with regard to jurisdictional claims in published maps and institutional affiliations.
Ready to submit your research? Choose BMC and benefit from:

- fast, convenient online submission

- thorough peer review by experienced researchers in your field

- rapid publication on acceptance

- support for research data, including large and complex data types

- gold Open Access which fosters wider collaboration and increased citations

- maximum visibility for your research: over $100 \mathrm{M}$ website views per year

At BMC, research is always in progress.

Learn more biomedcentral.com/submissions 